\title{
A SURVEY OF NURSES' OPINIONS ABOUT DELIRIUM IN ICU
}

Nikeshmani Rajbhandari $1^{*}$, Naba Raj Koirala ${ }^{2}$, Lalit Kumar Rajbanshi ${ }^{3}$, Batsalya Arjyal ${ }^{4}$

\section{Affiliation}

1. Lecturer, Department of Psychiatry and Mental Health, Birat Medical College and Teaching Hospital, Nepal.

2. Professor \& Head, Department of Psychiatry and Mental Health, Birat Medical College and Teaching Hospital, Nepal

3. Associate Professor, Department of Anaesthesia and Critical Care, Birat Medical College and Teaching Hospital, Nepal

4. Assistant Professor, Department of Anaesthesia and Critical Care, Birat Medical College and Teaching Hospital, Nepal

\section{ARTICLE INFO}

Received : 15 June, 2021

Accepted : 15 July, 2021

Published : 04 November 2021

(C) Authors retain copyright and grant the journal right of first publication with the work simultaneously licensed under Creative Commons Attribution License CC - BY 4.0 that allows others to share the work with an acknowledgment of the work's authorship and initial publication in this journal.

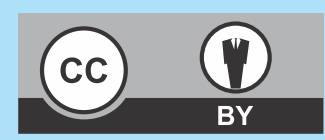

ORA 266

\section{DOI: https://doi.org/10.3126/bjhs.v6i2.40355}

\section{* Corresponding Author}

Dr. Nikeshmani Rajbhandari Lecturer

Department of Psychiatry and Mental Health Birat Medical College and Teaching Hospital, Nepal

Email: rajbhandarinikesh@gmail.com ORCID: $h$ ttps://orcid.org/0000-0003-0848-6298

\section{Citation}

Nikeshmani Rajbhandari, Naba Raj Koirala, Lalit Kumar Rajbanshi, Batsalya Arjyal. A survey of nurses' opinions about delirium in ICU. BJHS 2021;6(2)15. 1540-1544.

\section{ABSTRACT \\ Introduction}

Delirium is under diagnosed especially in ICU. In a study done in the Netherlands investigators found not only ICU nurses but also intensivists had difficulty in diagnosing delirium. With the impact that delirium has on patients' health, its monitoring and detection should be considered an integral part of patient care in critical care medicine, Knowledge and attitude are one of the primary unmet needs regarding early detection and treatment of delirium. Since nurses are the first line assessors in any ward we would like to carry out this cross-sectional survey on a group of nurses.

\section{Objective}

To assess the nurses' knowledge about delirium \& to compare the knowledge between those that have worked in ICU or critical care to those who haven't.

\section{Methodology}

This was a cross-sectional survey carried out at Birat medical college and teaching hospital. Here all nurses were requested to fill a questionnaire that consisted of 15 questions. A time-frame of 1 week was taken to gather the data.

\section{Result}

We collected data from 124 nurses, who had varying educational degrees, Eighty-four were currently working in an ICU and $32.5 \%$ of those not working had previously worked in an ICU. Over all knowledge level for nurses was poor where 94 of 124 nurses had less than 50\% scores. About $27 \%$ of those who had worked in an ICU setting had good knowledge compared to $17.5 \%$ of those who had not.

\section{Conclusion}

Training regarding delirium is required to increase identification and adequate management to decrease morbidity due to delirium.

\section{KEYWORDS}

Delirium, ICU, nurses' knowledge 


\section{INTRODUCTION}

Delirium is an acute brain dysfunction characterized by cognitive impairment, that is fluctuating, and disturbance in consciousness. ${ }^{1}$ It has many etiologies, all of which result in a similar pattern of symptoms and signs, which are related to cognition and consciousness. Usually delirium has a rapid onset and a brief and fluctuating course, with rapid improvement on removal of causative factor. It is a common condition and affects about $15 \%$ of patients in general medical and surgical wards. ${ }^{2}$ Though the incidence of delirium is up to $40 \%$ to $60 \%$ in the ICU it is an under diagnosed condition in intensive care unit or critical care unit. Lack of awareness about the need to diagnose and treat delirium is probably the major factor. ${ }^{2,3}$ Delirium causes burden to the hospital and society. It increases hospital mortality by three folds, while also increasing length of stay in the ICU and consequently increases hospital stay. ${ }^{3}$ Association between delirium and early deaths, functional disability, admission to residential care, and increased burden to caregivers have also been seen. ${ }^{3,4}$ With delirium having such a huge impact on patients there is a budding conscience that it is a public health problem. Data have shown that even though most health care workers deem delirium as an important health issue, few actually monitor it, are able to detect it and hence it remains largely under diagnosed. ${ }^{4}$

With the impact that delirium has on patients' health, its monitoring and detection should be considered as an integral part of patient care in critical care medicine, where the prevalence of delirium is highest. ${ }^{5}$ There should be guidelines in place in all ICU's around the country to monitor delirium. ${ }^{5}$ But for implementation of such guidelines and hence its success, it is important to know the views of healthcare workers about delirium. Knowledge and attitude are one of the primary unmet needs regarding early detection and treatment of delirium. Since nurses are the first line assessors in any ward we would like to carry out this cross-sectional survey on a group of nurses.

In a study done in the Netherlands investigators found not only ICU nurses but also intensivists had difficulty in diagnosing delirium. Without help of reliable, validated diagnostic tool only $35 \%$ of ICU delirium were recognized by nurses. ${ }^{1}$ A Swedish nation-wide survey of ICU head-nurses of the 58 respondents only one reported using a validated delirium screening tool and almost half reported using clinical judgement to diagnose delirium. The study also concluded that awareness of delirium is low and most centers lacked use of validated tools for delirium screening. ${ }^{2}$ Another survey carried out amongst 912 health professional about their opinions of delirium observed though most of them considered delirium to be a common and serious problem that was under diagnosed, few actually took steps to monitor the problem. ${ }^{3}$ In comparison to altered level of consciousness, presence of pain, or improper placement of an invasive device only $3 \%$ of 331 nurses from 16 different intensive care units ranked delirium as most important condition to evaluate. ${ }^{5}$ A simple educational intervention can improve the capacity of ICU nurses in detecting delirium early. ${ }^{6}$ Hence through this study we wished to assess the nurses' knowledge about delirium and additional to compare the knowledge between those that have worked in ICU or critical compared to those who haven't.

\section{METHODOLOGY}

This was a cross-sectional survey carried out at Birat Medical College and Teaching Hospital. A total of 200 nurses who worked at Birat Medical College and Teaching Hospital, were requested to fill a questionnaire that consisted of 15 questions. The questionnaire was developed by the authors following an iterative process of item generation, construction and refinement. Sample size was calculated assuming views of the nurses comes true as $50 \%$. Using the formula $n=\left(Z^{2} p^{*} q\right) / d^{2}$, where $d$ was considered as absolute difference, and considering for $20 \%$ non-response we calculated a sample size of 116 . A time-frame of 1 week was taken to gather the data, during which those that responded were included in the study. Statistical analysis was using SPSS v.22.

\section{RESULTS}

We collected data from 124 nurses, who had varying educational degrees, rest did not want to participate in the study. There was only one participant who had completed Masters in Science (Nursing) and most (72\%) of the participants had a degree in certificate level nursing, the rest (27\%) had received a bachelor's degree in nursing. The mean age of participants was $23.96 \pm 4.224$ years, with minimum age being 19 years and maximum 50 years. Almost $64 \%$ (79) of the participants were between $21-25$ years of age, 23 (18.5\%) between 26-30 years, 17 (13.7\%) were 20 years or less and 5 (4\%) were 31 years or more.

Among the 124 nurses about $89 \%$ had been working since 15 years, minimum duration of work experience being 1 month and maximum 20 years with a mean duration of $2.617 \pm 3.2$ years.

Eighty-four (67.7\%) participants said that they currently worked in an intensive care unit (ICU)or critical care unit as compared to the 40 that did not. Of those who were not presently working in an ICU or critical care, $32.5 \%$ reported that they had previously worked in such a setting. More than $74 \%$ of the participants said that they had not received any specific training on delirium and only $43 \%$ participants said that delirium was regularly screened at their centre $31 \%$ said it was not screened for and $25 \%$ did not know whether delirium was screened at their place of work. Of the 84 participants who were presently working at a ICU or critical care setting 2 did not answer the question, about $54 \%$ said it was screened for, $33 \%$ said it wasn't screened for and $11 \%$ said they did not know whether or not patients were screened for delirium. Amongst these 84 participants only $22.6 \%$ said they had any training or education regarding delirium.

Regarding opinion about delirium, more than $51 \%$ believed it was under diagnosed, $40 \%$ thought it was adequately diagnosed and about $9 \%$ did not respond. Similarly, results for other questions are stated in Table 1. 
Table 1: Frequency distribution in percentage of Opinion about Delirium

\begin{tabular}{|c|l|c|c|}
\hline S.No. & Question & Yes (\%) & No (\%) \\
\hline 1. & "Delirium is normal part of ICU admission & 62 & 38 \\
\hline 2. & "Delirium causes increased hospital stay" & 65 & 33 \\
\hline 3. & $\begin{array}{l}\text { "Regular monitoring of Delirium is not } \\
\text { necessary" }\end{array}$ & 8 & 92 \\
\hline 4. & $\begin{array}{l}\text { "Delirium is a Mental illness with no } \\
\text { physical aetiology" }\end{array}$ & 48 & 52 \\
\hline 5. & $\begin{array}{l}\text { "Delirium impacts weaning from } \\
\text { mechanical ventilation" }\end{array}$ & 56 & 42 \\
\hline 6. & $\begin{array}{l}\text { "Delirium is self-resolving and requires no } \\
\text { active treatment" }\end{array}$ & 42 & 56 \\
\hline 7. & $\begin{array}{l}\text { "Patient's with delirium should be } \\
\text { restrained all the time" }\end{array}$ & 16 & 84 \\
\hline
\end{tabular}

Of the 11 questions that were posed to the participants 3 were multiple choice questions where they were allowed to choose more than one option.

Table 2: Frequency distribution and percentage of different Medications used for delirium in ICU or critical care as answered by participants

\begin{tabular}{|c|l|c|c|}
\hline S.N. & Medication & $\begin{array}{c}\text { Medication } \\
\text { used }\end{array}$ & Percentage \\
\hline 1. & Haloperidol & 75 & 60.5 \\
\hline 2. & Olanzapine & 19 & 15.3 \\
\hline 3. & Lorazepam & 66 & 53.2 \\
\hline 4. & Quetiapine & 3 & 2.4 \\
\hline 5. & Diazepam & 51 & 41.1 \\
\hline 6. & Midazolam & 49 & 39.5 \\
\hline 7. & Dexmedetomidine & 18 & 14.5 \\
\hline
\end{tabular}

More than $70 \%$ reported chronic alcohol use was a risk factor for delirium, only $13 \%$ reported baseline hypertension to be a risk factor. At the same time 54\% thought pre-existing dementia as a risk factor for delirium. Thirty-nine percent thought benzodiazepine use and only $24 \%$ said pain could be a risk factor

Table 3: Frequency distribution of Different symptoms of Delirium chosen by participants.

\begin{tabular}{|l|l|c|c|}
\hline S.No. & \multicolumn{1}{|c|}{ Symptoms of Delirium } & N & $\%$ \\
\hline 1. & Impaired consciousness \& attention & 80 & 64.5 \\
\hline 2. & Cognitive disturbances & 89 & 71.8 \\
\hline 3. & Psychomotor disturbances & 42 & 33.9 \\
\hline 4. & Disturbed Sleep-Wake cycle & 39 & 31.5 \\
\hline 5. & Violence & 47 & 37.9 \\
\hline
\end{tabular}

As shown in Table 3 Most participants agreed that impaired consciousness and attention (65\%) and cognitive disturbances (72\%) were symptoms of delirium. Though not a criteria listed in the ICD-10 as a symptom of delirium, almost $38 \%$ of nurses selected violence as symptom. Knowledge level of nurses regarding delirium:
To quantify the level of knowledge of the participants we allocated one point for each correct answer for all single choice questions and for multiple choice questions we allocated one point for all correct options. Those who secured more than $50 \%$ correct answers were considered to have good knowledge. When considering only the single option questions $38 \%$ revealed good knowledge about delirium. Regarding risk factors for delirium almost $71 \%$ of participants scored less than $50 \%$, which reflected that the remaining $29 \%$ had good knowledge. For the question about symptoms of delirium more than $72 \%$ had less than $50 \%$ score. Approximately, $76 \%$ had poor knowledge about delirium.

A chi-square test was performed to examine the relation between working experience in ICU and level of knowledge about delirium. The relation between these variables was not significant, $\chi^{2}(1, N=124)=1.44, p=.23$. We also examined the statistical relationship between educational level and knowledge regarding delirium. The relation between these variables was also not significant, $\chi^{2}(1, N=124)=1.403, p=.24$.

Table 4: Distribution of knowledge level of those presently working or not in ICU or critical care

\begin{tabular}{|c|c|c|c|}
\hline S.No. & Working in ICU & \multicolumn{2}{|c|}{ Knowledge Level } \\
\hline & & $<50 \%$ & $>50 \%$ \\
\hline 1. & Yes & 61 & 23 \\
\hline 2. & No & 33 & 7 \\
\hline
\end{tabular}

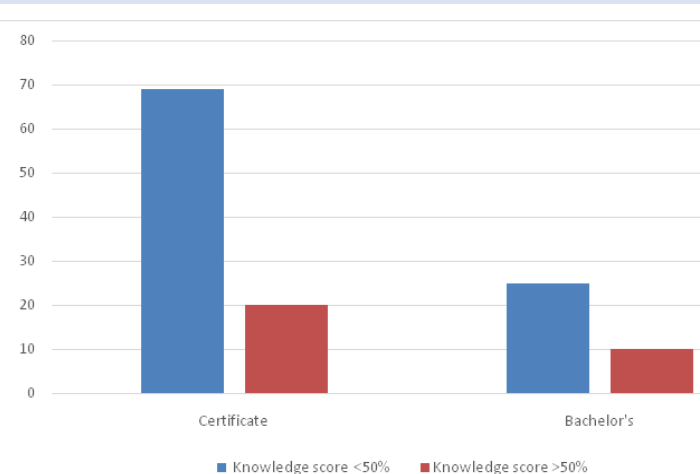

Figure 1: Level of education and knowledge score

\section{DISCUSSION}

In our study 124 nurses' participated, most of whom were about 24 years of age, mostly were educated up to certificate level nursing with an average working experience of 2.6 years. Of the 124 participants $68 \%$ were currently working in at a ICU or critical care unit, and of those who were not presently working at such a unit,about 32\% had previously worked at such a setting. More than $74 \%$ did not receive any training in delirium, and there was no mechanism for screening of delirium at work places of $32 \%$ of the participants and $25 \%$ said they were not aware whether it was screened for. Even though the society of critical care medicine sedation and analgesia guidelines considers assessment of delirium in the ICU an integral part 
of adult patient monitoringour findings are in agreement with a web-based American survey among 331 nurses, where only $40 \%$ stated that even though their units had a sedation protocol it did not specify that delirium should be assessed or they were not sure if the protocol did specify it. ${ }^{7}$ In this study the mean age of the respondents was 40.2 years with and average working experience of 13.6 years. ${ }^{5}$ Even with a very low mean age of participants and a much less duration of experience among participants in our study this finding was comparable. Another American survey, and an Australian study also had similar findings. ${ }^{3,8}$ Reports from a Swedish survey stated that $62 \%$ of the 82 ICUs used a method for observation of delirium. ${ }^{2}$ The Swedish study questionnaire was completed by ICU head nurses, among them $54 \%$ said delirium was screened for in their workplace. One meta-analysis showed in an intensive care unit nearly a third of patients developed delirium and these patients had longer stays in hospital and were at increased risk of death during admission and cognitive impairment following discharge. ${ }^{4}$ Even with these risks delirium is severely under-diagnosed, especially in the ICU. ${ }^{1,2,4}$ Without the help of validated, reliable and easy-to-use diagnostic instrument both ICU nurses as well as intensivists have difficulty in diagnosing presence of delirium. ${ }^{1}$ Almost two-thirds of participants in this study said they did not have any training in screening for delirium. A short composite and simple educational intervention in nurses, was seen to improve the capacity of ICU nurses to detect delirium. ${ }^{5}$

Delirium is under diagnosed but only $51 \%$ of the participants agreed to this and $40 \%$ believed it was adequately diagnosed, this is in contrast to a study done in Ireland where more than 93\% acknowledged that delirium was an under diagnosed syndrome. ${ }^{9}$ This discrepancy could be because the Irish study was among intensive care nurses' and $84 \%$ said they had sedation protocol but the protocols did not assess for delirium. This indicates that exposure to more cases of delirium can increase knowledge regarding it. The top three risk factors for delirium was chronic alcohol use, pre-existing dementia and benzodiazepine use. Dementia and alcohol use was thought to be major contributing factors for risk of delirium by participants of other studies as well. ${ }^{2,3,9}$

Even though almost two-third of participants correctly identified cognitive disturbances and impaired consciousness and attention as symptoms of delirium less than one-third identified the two symptoms. At the same time more than $75 \%$ had poor knowledge about delirium. This indicates there is a dire lack of education regarding delirium despite a growing recognition that it is a major problem in hospitals and especially in ICU. Less than half of the participants said that delirium was screened at their workplace even though this is more than the $16 \%$ stated in another study, there was a severe lack of knowledge among our participants about delirium. ${ }^{9}$ Although delirium was believed to cause many negative outcomes in patients such as increased mortality, cognitive impairment, delayed weaning from ventilator and increased time spent in ICU, only $4 \%$ had thought it was important to monitor for delirium. ${ }^{2,4,8}$ The notion that delirium is difficult to monitor in the ICU because patients remain sedated most of the time, seems to be oversimplified. ${ }^{6}$ Using delirium specific assessment tools ICU health-workers, who encounter most cases of delirium can diagnose presence of delirium and hence monitor for progress with great ease.

\section{CONCLUSION}

Delirium is an underdiagnosed condition, even though it is a major cause for mortality and increased morbidity especially among ICU patients. Knowledge about delirium was found to be low in our study. From various studies, including ours we see that there is a need for increasing education and training about delirium.

\section{RECOMMENDATIONS}

We have seen from our study, there is no protocol for screening of delirium at the centre where our participants work. This causes, as also believed by the participants, under-diagnosis. A common speculation of the past that patients in ICU remain sedated mostly appears to be a major hindrance for adequate assessment of delirium. ${ }^{6}$ These problems could be overcome by providing training to assess for delirium by usage of standardized screening tools. Setting up evidence based protocols to assess for delirium with the help of standardised screening tools could be beneficial for health-workers as well as for patients.

\section{LIMITATIONS OF THE STUDY}

As with majority of studies this study is also subject to limitations. This study was carried out in a single centre, and opinion was gathered via a subjective questionnaire that was not validated prior to conducting the study, among nurses selected through purposive sampling. Incorporating, opinions from nurses working in different hospitals at different cities of Nepal and randomly selecting the locations would have been better. Also using a validated questionnaire and conducting the study amongst not just nurses but other health-care workers would give us a better picture of the status of knowledge of delirium among health professionals around the country. This would in turn help us design better studies to help us create evidence based protocols for assessment of delirium in our patients.

\section{ACKNOWLEDGEMENT}

We would like to thank the management of Birat Medical College \& Teaching Hospital for allowing us to conduct this study.

\section{CONFLICT OFINTEREST}

None

\section{FINANCIAL DISCLOSURE}

None 


\section{REFERENCES}

1. Spronk PE, Riekerk B, Hofhuis J, Rommes JH. Occurrence of delirium is severely underestimated in the ICU during daily care. Intensive care medicine. 2009 Jul 1;35(7):1276-80DOI: 10.1007/s00134-009-1466-8

2. Forsgren LM, Eriksson M. Delirium-awareness, observation and interventions in intensive care units: a national survey of Swedish ICU head nurses. Intensive and Critical Care Nursing. 2010 Oct 1;26(5):296-303.DOI: 10.1016/j.iccn.2010.07.003

3. Ely EW, Stephens RK, Jackson JC, Thomason JW, Truman B, Gordon S, Dittus RS, Bernard GR. Current opinions regarding the importance, diagnosis, and management of delirium in the intensive care unit: a survey of 912 healthcare professionals. Critical care medicine. 2004 Jan 1;32(1):106-12. DOI: 10.1097/01.CCM.0000098033.94737.84

4. Salluh JI, Wang H, Schneider EB, Nagaraja N, Yenokyan G, Damluji A, Serafim RB, Stevens RD. Outcome of delirium in critically ill patients: systematic review and meta-analysis. bmj. 2015 Jun 3;350.DOI: 10.1136/bmj.h2538

5. Devlin JW, Fong JJ, Howard EP, Skrobik Y, McCoy N, Yasuda C, Marshall J. Assessment of delirium in the intensive care unit: nursing practices and perceptions. American Journal of Critical Care. 2008 Nov 1;17(6):555-65.PMID: 18978240

6. Devlin JW, Marquis F, Riker RR, Robbins T, Garpestad E, Fong JJ, Didomenico D, Skrobik Y. Combined didactic and scenario-based education improves the ability of intensive care unit staff to recognize delirium at the bedside. Critical Care. 2008 Feb;12(1):1-6.DOI: 10.1186/cc6793

7. Barr J, Fraser GL, Puntillo K, Ely EW, Gélinas C, Dasta JF, Davidson JE, Devlin JW, Kress JP, Joffe AM, Coursin DB. Clinical practice guidelines for the management of pain, agitation, and delirium in adult patients in the intensive care unit. Critical care medicine. 2013 Jan 1;41(1):263-306.DOI: 10.1097/CCM.0b013e3182783b72

8. Shehabi Y, Botha JA, Boyle MS, Ernest D, Freebairn RC, Jenkins IR, Roberts BL, Seppelt IM. Sedation and delirium in the intensive care unit: An Australian and New Zealand perspective. Anaesthesia and intensive care. 2008 Jul 1;36(4):570-9.DOI: 10.1177/ 0310057 X0803600423

9. Glynn L, Corry M. Intensive care nurses' opinions and current practice in relation to delirium in the intensive care setting. Intensive and Critical Care Nursing. 2015 Oct 1;31(5):269-75. DOI: 10.1016/ j.iccn.2015.05.001

\section{Questionnaire}

Age

Education level

How many years have you been working for?
1. Do you presently work in ICU or critical care?
a) Yes
b) No

2. Have you previously worked at an ICU?
a) Yes
b) No

3. Is delirium regularly screened for at the ICU where you work
a) Yes
b) No
c) Don'tknow

4. Have you received any education or training on delirium?
a) Yes
b) No

5. Do you think Delirium is underdiagnosed?
a) Yes
b) No

\section{Opinions about Delirium}

1. Delirium is a normal part of ICU admission
a) Yes
b) No

2. Delirium does not cause increased hospital stay
a) Yes
b) No

3. Regular monitoring for Delirium is not necessary.
a) True
b) False

4. Delirium is a mental illness and has no physical etiology
a) True
b) False

5. Does delirium impact weaning from mechanical ventilation?
a) Yes
b) No

6. Which drug is used in cases of delirium at your centre? [You can choose more than one]
a) Haloperidol
b) Olanzapine
c) Lorazepam
d) Quetiapine
e) Diazepam
f) Midazolam
g) Dexmedetomidine
h) Others

7. Active treatment of delirium is not necessary as it is self-resolving?
a) True
b) False

8. Which are risk factors for developing delirium? [you can choose more than one]
a) Pre-existing dementia
b) History of hypertension
c) Chronic Alcohol use
d) Benzodiazepine use
e. Pain
f) Others (please mention)

9. Patients with delirium should be restraint all the time
a) Yes
b) No

10. Which of the following are symptoms of delirium? [You can choose more than one option]

a) Impaired consciousness \& attention

b) Cognitive disturbances (problems in thought)

c) Psychomotor disturbances

d) Sleep-wake cycle

e. Violence

f. Others (please mention) 